\title{
Herniation after deep circumflex iliac artery flap: two cases of rare complication
}

\author{
Hee-Sung Kim ${ }^{1}$, Jae-Young Kim², Hyuk Hur ${ }^{3}$ and Woong Nam ${ }^{4^{*}}$ (D)
}

\begin{abstract}
Herniation after harvesting of deep circumflex iliac artery (DCIA) flap is a known but not a common complication. It occurs about 2.8 to $9 \%$ according to the literatures and can proceed to a more severe complication such as bowel obstruction. There are several factors that exacerbate the risk: surgical factors, operator factor, and patient factors. Surgical factors include large anatomical defect and denervation of related muscles. Operator factor stands for unpunctual suture technique. Patient factors represent obesity, diabetes, pulmonary disease, smoking habits, and so on. Thus, herniation might occur regardless of meticulous suture. Herein, we would like to report two cases of herniation after DCIA flap harvesting and repaired by Lichtenstein tension-free hernioplasty with literature review.
\end{abstract}

Keywords: Deep circumflex iliac artery (DCIA) flap, Hernia, Lichtenstein tension-free hernioplasty, Reconstruction, Bowel obstruction

\section{Background}

Deep circumflex iliac artery flap (DCIA) is first introduced by Taylor et al. in 1979 [1]. Since then, it has been used to reconstruct composite defect in the head and neck area. It provides similar contour of mandible and sufficient volume to accommodate implants than other osseous flaps [2]. However, several donor site complications were reported after DCIA flap such as damage of lateral cutaneous femoral nerve, gait disturbance, bowel obstruction, and herniation [3, 4].

Among them, herniation occurs from 2.8 to $9 \%$ of patients and can lead to a more severe condition such as bowel obstruction $[5,6]$. It may show various ranges of symptoms from acute to long-standing discomforts after several months or years of operation [7]. Such as a bulge in the groin, pain in the groin that may also include a heavy or dragging sensation, pain followed by tenderness, and nausea, vomiting, or fever caused by bowel obstruction. When patients complain of such abdominal pain, computed tomography (CT) scan is used for confirmation of diagnosis and operation will be performed by various methods including Lichtenstein tension-free technique $[8,9]$. When herniation occurs, we can consider

\footnotetext{
* Correspondence: OMSNAM@yuhs.ac

${ }^{4}$ Department of Oral and Maxillofacial Surgery, Oral Cancer Research Institute, Yonsei University College of Dentistry, Yonsei-ro 50, Seodaemun-gu, Seoul 120-752, Korea

Full list of author information is available at the end of the article
}

several methods like Bassini repair, Shouldice repair, and tension-free hernioplasty. Among them, tension-free hernioplasty is known as a representative method.

Herein, we present two cases of hernia formation after DCIA flap which were treated by Lichtenstein tension-free hernioplasty, cooperated with the Department of Surgery.

\section{Case presentation \\ Case 1}

A 53-year-old male visited the Department of Oral and Maxillofacial Surgery at the Dental Hospital. His chief complaint was swelling and pain on left mandibular molar about 5 months ago. There was no specific past medical history. But he was a heavy smoker. He smoked two packs of cigarettes per day during 10 years. His body mass index (BMI) was 27.04 (height $172 \mathrm{~cm}$, body weight $80 \mathrm{~kg}$ ). Left mandibular second premolar and first molar showed severe mobility accompanied by gingival swelling, pus discharge, and easy bleeding on probing. Incisional biopsy revealed invasive squamous cell carcinoma. Additional imaging studies including magnetic resonance imaging (MRI) and positron emission tomography (PET) were done. Osteolytic lesion and enlarged lymph nodes in the left level II with intense fluorodeoxyglucose (FDG) uptake were observed on the PET image. Thus, operation was performed under general anesthesia as follows: wide excision, segmental mandibulectomy, modified radical neck dissection, and

\section{Springer}

(c) 2016 Kim et al. Open Access This article is distributed under the terms of the Creative Commons Attribution 4.0 International License (http://creativecommons.org/licenses/by/4.0/), which permits unrestricted use, distribution, and reproduction in any medium, provided you give appropriate credit to the original author(s) and the source, provide a link to the Creative Commons license, and indicate if changes were made. 
reconstruction of hard tissue defect with DCIA flap harvested from the right side. DCIA flap was harvested with internal oblique muscle. Layer-by-layer suture was carried out precisely to prevent herniation. Unfortunately, however, 2 months after surgery, he visited our department with dull pain on flap harvesting site when palpated. He was referred to Department of Surgery for further evaluation and proper management. He was diagnosed as incisional hernia on the right flank area after CT taking (Fig. 1). Periodic checkup and surgical treatment were planned if herniation becomes worse. After 5 months, he underwent operation because herniation with pain was getting worse. The operation was performed at Department of Surgery. We could observe the herniation of small bowel through the defect formed by DCIA flap harvesting and atrophic change of surrounding muscle and fascia (Fig. 2). Anterior abdominal muscles were sutured to adjacent periosteum with 2-0 Prolene. Mesh (Bard ${ }^{\text {ma }}$ mesh, $10 \times 14$ in., Bard Davol Inc., Warwick, USA) was applied over and sutured with the surrounding structures (Fig. 3). Eight months after herniorrhaphy, he complained of dull pain of the operation site and right lower flank area protrusion. Department of Surgery diagnosed recurrence of herniation, and patient was diagnosed as recurrence of herniation. He underwent muscle approximation with layer-by-layer suture which was the same method used at the previous operation. It has shown a satisfactory result during 7 months to date without any recurrence.

\section{Case 2}

A 52-year-old male underwent segmental mandibulectomy and reconstruction of the defect with DCIA flap due to recurrent invasive squamous cell carcinoma. There was no specific past medical history. But he was a heavy smoker. A $6.5 \times 3.0 \mathrm{~cm}$-sized DCIA flap was harvested with internal oblique muscle. Peritoneum was not

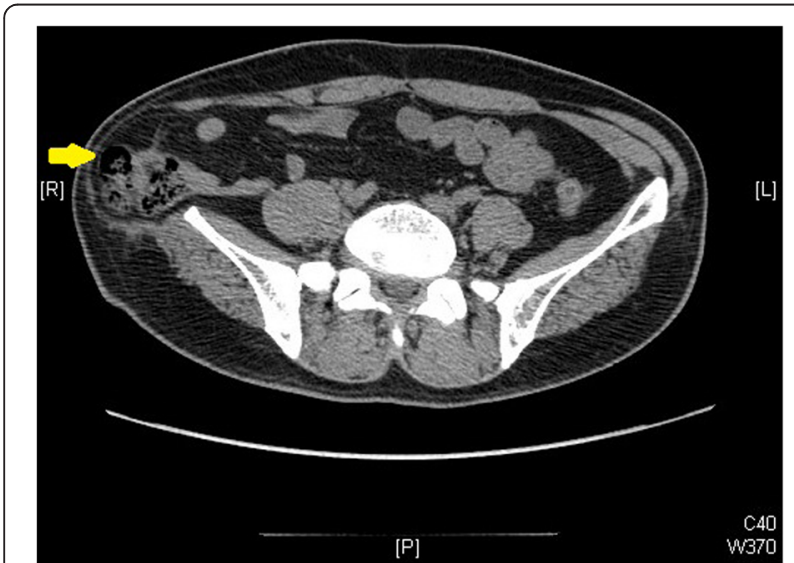

Fig. 1 CT image (case 1). Herniation (arrow) can be observed through bone defect caused by DCIA flap harvesting. There are no anatomical barriers to avoid herniation

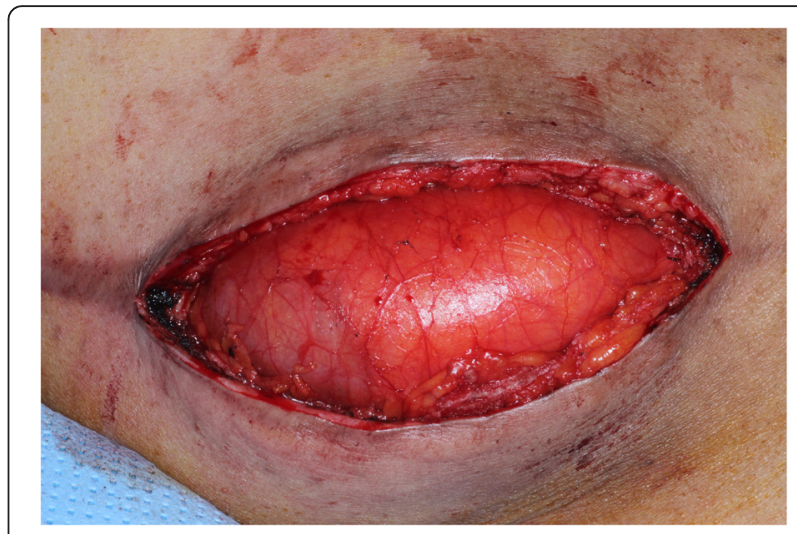

Fig. 2 Clinical photograph (case 1). Herniated small bowel and atrophic change of the surrounding muscle and fascia

exposed during flap harvesting. Meticulous suture was performed as usual.

After 6 months, the patient complained of dull pain on DCIA flap donor site. We suspected herniation and consulted to Department of Surgery for further evaluation. The patient was diagnosed as incisional ventral hernia. We could find the herniated bowel through iliac bone defect on CT image (Fig. 4). After 4 months (11 August 2014), operation for repair of ventral hernia was performed by general surgeons. Hernia sac was identified on the bony defect area formed by the previous surgery (Fig. 5). The transverse abdominis muscle wall became thin with atrophic change. After excision of herniated sac, the peritoneum was closed by continuous suture. The transverse abdominis muscle was plicated and fixed on iliac crest periosteum to reinforce weakened muscle layer. Then, mesh $\left(\right.$ Bard $^{\text {Tim }}$ mesh $10 \times 14$ in., Bard Davol Inc., Warwick, USA) was applied over the transverse abdominis muscle. Interrupted suture was performed on internal oblique muscle. Finally, we confirmed that the weakened muscle area had disappeared and herniation was properly

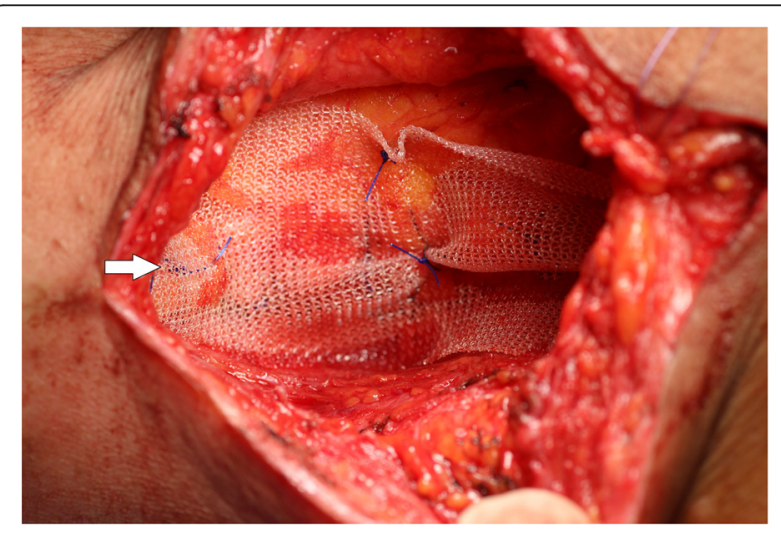

Fig. 3 Lichtenstein tension-free hernioplasty. Mesh (arrow) is applied over and sutured with surrounding structures 


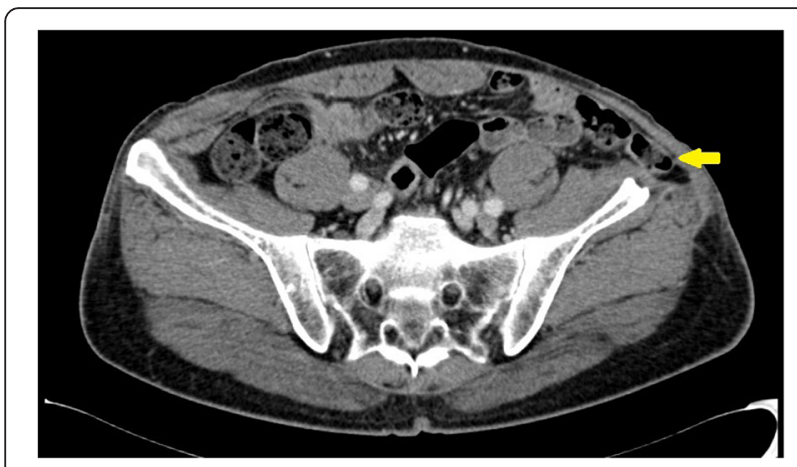

Fig. 4 CT image (case 2). Herniation (arrow) can be observed through the bone defect caused by DCIA flap harvesting. There are no anatomical barriers to avoid herniation

repaired. Fifteen months have passed until now without any recurrence of herniation.

\section{Discussion}

DCIA flap is a preferable reconstructive method after segmental maxillectomy or mandibulectomy. It is possible to reconstruct the defect area as well as bring functional and esthetic results $[10,11]$. The bony stock and height is proper for osteointegration of dental implant and bearing bite force [12]. Reshaping is not necessary during surgery due to its original anatomical shape. Hairless skin on the groin area is suitable for re-coverage of intraoral mucosa [11]. Rogers et al. reported better quality of life and lesser donor site morbidity when DCIA flap was used compared with fibula-free flap [13].

Although DCIA flap has several advantages, donor site morbidities such as gait disturbance, lateral femoral cutaneous nerve damage, and herniation make it difficult for the patients as well as the operators. Especially, occurrence of herniation after several months or years of operation makes it difficult for the operator to manage the patient as he needs additional surgery [7]. According

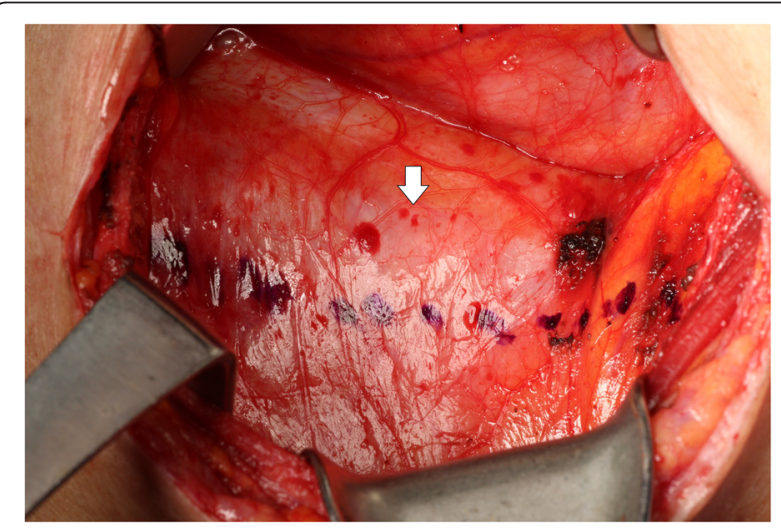

Fig. 5 Clinical photograph (case 2). Herniated sac (arrow) is identified through the bone defect caused by the previous surgery to the literature, the incidence is reported from 2.8 to $9 \%[5,6]$. The patients complain acute abdominal discomfort or long-term pain and an abdominal mass showing internal noise at auscultation as well as palpation [8]. CT image is used for definite diagnosis [8]. In these cases, herniation was also developed at 2 and 6 months after surgery with dull pain on the donor site. Sometimes, pelvic bone fracture can occur after harvesting of DCIA flap or iliac bone graft. It is especially true in case of osteopenic conditions and operations including anterior iliac crest [14] .At first, we also suspected fracture of pelvic bone because of their obesity which has adverse effects on healing process. Especially, it was more suspicious in case 1 , because his heavy weight could bring more pressure on pelvic bone and only 2 months had passed after surgery. However, the impression was herniation due to several reasons. They were not osteopenic conditions, and anterior-superior iliac spine was preserved more than $3 \mathrm{~cm} \mathrm{[14].} \mathrm{The} \mathrm{patient} \mathrm{in}$ case 1 had seldom walked during 2 months because he underwent several other operations. After CT taking, definite diagnosis as herniation was made.

A number of reasons were suggested for herniation at a donor site. First, anatomical defect is related. DCIA flap is harvested with the surrounding muscles to maintain blood supply. Defect of the surrounding muscles causes loss of anatomical barrier, which brings herniation when managed improperly [15]. The flap must include the skin, external oblique, internal oblique, and transverse abdominis muscle to keep the perforators. This makes bigger defect size and raises the possibility for herniation. Forrest et al. reported the incidence was $12 \%(7 / 59)$ among osteocutaneous flaps, whereas the incidence was $4 \%$ (1/23) among osseous flaps [16]. Second, we consider the general conditions of the patient such as history of chronic obstructive pulmonary disease, obesity, diabetes, gender, constipation, and smoking habits [4, 17]. Especially, obese patients have greater risk of incisional hernia due to increased intra-abdominal pressure [18]. Other perioperative factors including inadequate analgesia, vomiting, and postoperative pneumonia are also considered [4]. Furthermore, the risk of herniation may be exacerbated by denervation of rectus muscle [3]. Internal oblique muscle harvesting may bring this denervation through interruption of motor nerve supply [19]. We can prevent this denervation with precise dissection of the nerves, which lie between the internal oblique and transverse abdominal muscles [19]. In these cases, we consider that the primary reason is improper management of the surrounding muscles. Besides, we take harvesting of the internal oblique muscle, obesity, and previous heavy smoking habits into consideration.

Surgical techniques and materials have been proposed to prevent or minimize hernia formation. First of all, 
surgeons ought to make an effort to restructure the abdominal wall. Meticulous suturing of the transversalis fascia to the iliacus fascia, and all three layers of the abdominal muscles to the tensor fascia latae and gluteus medius are necessary [16]. Apart from the primary closure technique, Halsnad et al. suggested the use of titanium plate for primary reconstruction of the osseous defect [7]. In 2007, Iqbal et al. reported insertion of titanium coils (Protack ${ }^{\mathrm{mu}}$, US Surgical Corp.) to attach Prolene (polypropylene) mesh into the cancellous and cortical bone of the donor site [15]. Despite of these efforts, however, herniation through DCIA flap occurs [4]. We can consider various open surgery techniques such as Bassini repair, Shouldice repair, and tensionfree hernioplasty when herniation occurs [20]. Among them, Lichtenstein tension-free hernioplasty is one of the representative methods for hernia repair using polypropylene mesh [21]. It was developed in 1984 and was improved through 1988 [22]. Although some disadvantages were reported such as increasing stiffness of the abdominal wall and abdominal pressure, American College of Surgeons considers this method as a gold standard in hernia repair [22-24].

\section{Conclusions}

Herniation after DCIA flap is a known complication but uncommon. Precise primary suture is always important to prevent herniation. Especially, it is more so when the patient is obese, a heavy smoker, and when the flap contains more muscles including internal oblique muscles. Also, careful observation and follow-up of the donor site, which is the groin area, should be done as well at the recipient site after DCIA flap. If pain or bulging is observed, patient should be referred to the Department of Surgery for evaluation on hernia. And if hernia occurs, repair using Lichtenstein tension-free hernioplasty should be performed at a proper period.

\section{Consent}

Written informed consent was obtained from the patient for publication of this case report and any accompanying images. A copy of the written consent is available for review by the Editor-in-Chief of this journal.

\section{Competing interests}

The authors declare that they have no competing interests.

\section{Authors' contributions}

$\mathrm{HH}$ surgically treated the two case of ventral hernia. NW operated as follows: wide excision, segmental mandibulectomy, modified radical neck dissection, reconstruction of hard tissue defect with DCIA flap, and harvested the DCIA flap. And he gave important input and carefully reviewed the manuscript. KHS and KJY wrote the manuscript and contributed significantly to the treatment of the patient. All authors read and approved the final manuscript.

\section{Author details}

${ }^{1}$ Department of Oral and Maxillofacial Surgery, Yonsei University College of Dentistry, Yonsei-ro 50, Seodaemun-gu, Seoul 120-752, Korea. ${ }^{2}$ Department of Oral and Maxillofacial Surgery, Gangnam Severance Hospital, Yonsei University College of Dentistry, Seoul, Korea. ${ }^{3}$ Department of Surgery, Yonsei University College of Medicine, Seoul, Korea. ${ }^{4}$ Department of Oral and Maxillofacial Surgery, Oral Cancer Research Institute, Yonsei University College of Dentistry, Yonsei-ro 50, Seodaemun-gu, Seoul 120-752, Korea.

Received: 20 December 2015 Accepted: 9 February 2016

Published online: 25 February 2016

\section{References}

1. Taylor GI, Townsend P, Corlett R (1979) Superiority of the deep circumflex iliac vessels as the supply for free groin flaps. Clinical work. Plast Reconstr Surg 64:745

2. Beahm KE, Hanasono MM, Shenaq S (2009) Iliac flap. In: Wei FC, Mardini S (eds) Flaps and reconstructive surgery. Elsevier, p 338

3. Hartman EH, Spauwen PH, Jansen JA (2002) Donor-site complications in vascularized bone flap surgery. J Invest Surg 15:185

4. Tan NC, Brennan PA, Senapati A et al (2009) Bowel obstruction following deep circumflex iliac artery free flap harvesting. $\mathrm{Br} J$ Oral Maxillofac Surg 47:645

5. Kantelhardt T, Stock W, Stützle H et al (1999) Results at the donor site after free microvascular iliac crest transplantation. Eur J Plast Surg 22:366

6. Duncan MJ, Manktelow RT, Zuker RM et al (1985) Mandibular reconstruction in the radiated patient: the role of osteocutaneous free tissue transfers. Plast Reconstr Surg 76:829

7. Halsnad SM, Dhariwal DK, Bocca AP et al (2004) Titanium plate reconstruction of the osseous defect after harvest of a composite free flap using the deep circumflex iliac artery. Br J Oral Maxillofac Surg 42:254

8. Valentini V, Gennaro P, Aboh IV et al (2009) Iliac crest flap: donor site morbidity. J Craniofac Surg 20:1052

9. Wysocki A, Kulawik J, Pozniczek M et al (2006) Is the Lichtenstein operation of strangulated groin hernia a safe procedure? World J Surg 30:2065

10. Urken ML, Weinberg H, Vickery C et al (1991) The internal oblique-iliac crest free flap in composite defects of the oral cavity involving bone, skin, and mucosa. Laryngoscope 101:257

11. Brown JS (1996) Deep circumflex iliac artery free flap with internal oblique muscle as a new method of immediate reconstruction of maxillectomy defect. Head Neck 18:412

12. Baliarsing AS, Kumar W, Malik NA et al (2010) Reconstruction of maxillectomy defects using deep circumflex iliac artery-based composite free flap. Oral Surg Oral Med Oral Pathol Oral Radiol Endod 109, e8

13. Rogers SN, Lakshmiah SR, Narayan B et al (2003) A comparison of the long-term morbidity following deep circumflex iliac and fibula free flaps for reconstruction following head and neck cancer. Plast Reconstr Surg 112:1517

14. Covani U, Ricci M, Santini S et al (2013) Fracture of anterior iliac crest following bone graft harvest in an anorexic patient: case report and review of the literature. J Oral Implantol 39:103

15. Iqbal M, Lloyd CJ, Paley MD et al (2007) Repair of the deep circumflex iliac artery free flap donor site with Protack (titanium spiral tacks) and Prolene (polypropylene) mesh. Br J Oral Maxillofac Surg 45:596

16. Forrest C, Boyd B, Manktelow R et al (1992) The free vascularised iliac crest tissue transfer: donor site complications associated with eighty-two cases. Br J Plast Surg 45:89

17. Lyons AJ, James R, Collyer J (2005) Free vascularised iliac crest graft: an audit of 26 consecutive cases. Br J Oral Maxillofac Surg 43:210

18. Sugerman HJ, Kellum JM Jr, Reines HD et al (1996) Greater risk of incisional hernia with morbidly obese than steroid-dependent patients and low recurrence with prefascial polypropylene mesh. Am J Surg 171:80

19. Urken ML (1995) lliac crest osteocutaneous and osteomusculocutaneous. In: Urken ML, Cheney ML, Sullivan MJ et al (eds) Atlas of regional and free flaps for head and neck reconstruction. New York, Raven Press, p 261

20. Mückter H, Reuters G, Vogel W (1994) Bassini and Shouldice repair of inguinal hernia. A retrospective comparative study. Chirurg 65(2):121-126

21. Lichtenstein IL, Shulman AG, Amid PK et al (1989) The tension-free hernioplasty. Am J Surg 157:188 
22. Peker K, Isik A, Inal A et al (2014) How Lichtenstein hernia repair affects abdominal and anal resting pressures: a controlled clinical study. Int J Clin Exp Med 7:363

23. Amid PK (2004) Lichtenstein tension-free hernioplasty: its inception, evolution, and principles. Hernia 8:1

24. Costello CR, Bachman SL, Ramshaw BJ et al (2007) Materials characterization of explanted polypropylene hernia meshes. J Biomed Mater Res B Appl Biomater 83:44

\section{Submit your manuscript to a SpringerOpen ${ }^{\circ}$ journal and benefit from:}

- Convenient online submission

- Rigorous peer review

- Immediate publication on acceptance

- Open access: articles freely available online

- High visibility within the field

- Retaining the copyright to your article

Submit your next manuscript at $>$ springeropen.com 